\title{
Autonomous Navigation of Hexapod Robots With Vision- based Controller Adaptation
}

\section{Conference Paper}

\section{Author(s):}

Bjelonic, Marko; Homberger, Timon; Kottege, Navinda; Borges, Paulo; Chli, Margarita (D; Beckerle, Philipp

\section{Publication date:}

2017

Permanent link:

https://doi.org/10.3929/ethz-a-010859391

Rights / license:

In Copyright - Non-Commercial Use Permitted

Originally published in:

https://doi.org/10.1109/ICRA.2017.7989655 


\title{
Autonomous Navigation of Hexapod Robots With Vision-based Controller Adaptation
}

\author{
Marko Bjelonic ${ }^{1 *}$, Timon Homberger ${ }^{2 *}$, Navinda Kottege ${ }^{3}$, Paulo Borges ${ }^{3}$, Margarita Chli $^{4}$, Philipp Beckerle ${ }^{5}$
}

\begin{abstract}
This work introduces a novel hybrid control architecture for a hexapod platform (Weaver), making it capable of autonomously navigating in uneven terrain. The main contribution stems from the use of vision-based exteroceptive terrain perception to adapt the robot's locomotion parameters. Avoiding computationally expensive path planning for the individual foot tips, the adaptation controller enables the robot to reactively adapt to the surface structure it is moving on. The virtual stiffness, which mainly characterizes the behavior of the legs' impedance controller is adapted according to visually perceived terrain properties. To further improve locomotion, the frequency and height of the robot's stride are similarly adapted. Furthermore, novel methods for terrain characterization and a keyframe based visual-inertial odometry algorithm are combined to generate a spatial map of terrain characteristics. Localization via odometry also allows for autonomous missions on variable terrain by incorporating global navigation and terrain adaptation into one control architecture. Autonomous runs on a testbed with variable terrain types illustrate that adaptive stride and impedance behavior decreases the cost of transport by $30 \%$ compared to a non-adaptive approach and simultaneously increases body stability (up to $88 \%$ on even terrain and by $54 \%$ on uneven terrain). Weaver is able to freely explore outdoor environments as it is completely free of external tethers, as shown in the experiments.
\end{abstract}

\section{INTRODUCTION}

Locomotion of mobile robots on rough terrain without prior information of the terrain structure is an important task. It is required for planetary exploration [1], missions in disaster zones [2], mining [3], and others. In this field, legged robots have gained increased attention, due to their mechanical ability to move on various types of challenging terrain [4].

Legged robots need to control the contact between the foot tips and the ground in order to ensure stability on rough terrain. Both reactive and proactive control approaches

1 M. Bjelonic is with the Robotic Systems Lab, ETH Zürich, 8092 Zürich, Switzerland and was with the Autonomous Systems Group, CSIRO, Pullenvale, QLD 4069, Australia at the time of this work.

2 T. Homberger is a student at the Department of Mechanical and Process Engineering, ETH Zürich, 8092 Zürich, Switzerland was with the Autonomous Systems Group, CSIRO, Pullenvale, QLD 4069, Australia at the time of this work.

${ }^{3}$ N. Kottege and P. Borges are with the Autonomous Systems Group, CSIRO, Pullenvale, QLD 4069, Australia. Correspondence should be addressed to navinda.kottege@csiro.au

${ }^{4}$ M. Chli is with the Vision for Robotics Lab, ETH Zürich, 8092 Zürich, Switzerland.

${ }^{5}$ P. Beckerle is with the Institute for Mechatronic Systems in Mechanical Engineering, Technische Universität Darmstadt, 64287 Darmstadt, Germany.

* These authors contributed equally to this work.

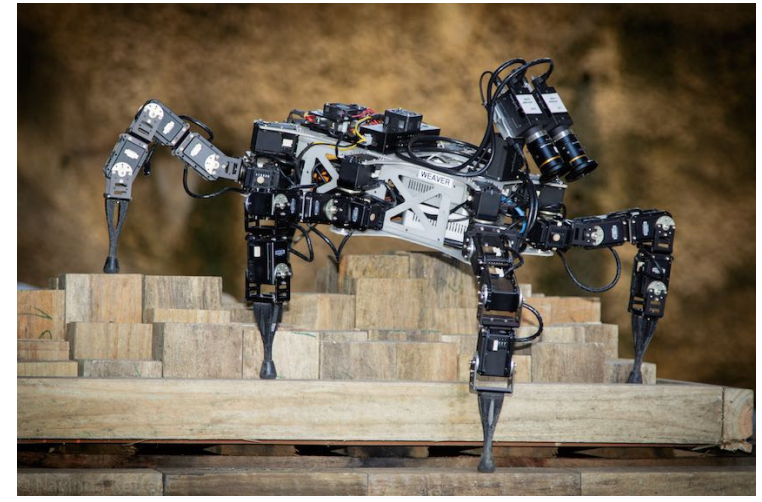

Fig. 1. The hexapod robot Weaver on the multi-terrain testbed.

have been used in the literature to perform leg adaptation to achieve this [5], [6], [7]. The former control approach reacts to ground collisions of the legs and adapts based on proprioceptive sensing. In contrast, a proactive approach avoids collisions by motion planning of all joints. However, previous investigations show that trajectory planning of the feet without adaptive execution is insufficient for walking robots in unstructured terrain due to unpredictable disturbances, such as bumps and slippage [8]. Autonomous operations in unknown terrain usually feature both reactive and proactive control architectures to achieve practical performance in realworld environments. The combination of these two control strategies is referred to as hybrid control in this work in accordance with [5].

The performance of legged robots highly depends on the chosen gait parameters. Moreover, the transition between different terrain types introduces conflicting objectives for the gait parameter choice. The results presented in [9] show the limitations of using constant gait parameters for maneuvering on diverse surface types. An elastic configuration of the leg yields benefits in efficiency and maneuverability of the robot on rough terrain. In contrast, the same leg configuration leads to high cost of locomotion on flat terrain. Parameter adaptation with respect to the characteristics of the ground is proposed to improve locomotion on varying terrain. Parameter adaptation of legged systems is also shown in [10] for a dynamic biped with a tether boom and for a quadruped in [11] with a focus on implementing a neural system model based on biological concepts.

This paper presents a hybrid control architecture that enables a legged robot to autonomously traverse uneven terrain and to adapt the gait parameters depending on the 
terrain characteristics. The robot's autonomy is achieved by using visual-inertial odometry on a custom built hardware setup. Moreover, the proposed controller is evaluated on a real hexapod robot, Weaver (Fig. 1). The hybrid controller introduces:

- Efficient and stable locomotion by adapting stride height, stride frequency and virtual stiffness.

- Exteroceptive terrain perception method for parameter adaptation of a reactive control architecture.

- Increased autonomy by combining a global navigation system with controller adaptation.

The paper is organized as follows: The hybrid controller is presented in Section III after introducing Weaver in Section II. The experimental results in Section IV are discussed by Section V and Section VI concludes the paper.

\section{HeXapod Platform Weaver}

Weaver was specially developed in-house for rough terrain traversal and introduced in [9]. One of the key differences to other hexapod robots is the five joints per leg. This allows efficient and stable locomotion on inclined surfaces, as the last link of the robot's leg can be aligned with the gravity vector. In addition, Weaver with its 30 Degrees of freedom (DoF) controls the body pose on inclinations to increase the Normalized Energy Stability Margin [12]. The direction of the gravity vector is determined by measurements of an Inertial Measurement Unit (IMU). The inclination controller, coupled with an impedance controller of the foot tip position in Cartesian space improves Weaver's locomotion on uneven terrain. It reacts to forces imposed by adapting the position of the foot tip.

The hybrid controller presented in this paper combines the exteroceptive terrain perception introduced in [13] with the proprioceptive controller introduced in [9] and implements an adaptation controller which adapts not only the leg stiffness but also the stride height and stride frequency. The following section further describes the hybrid control architecture.

\section{HYBRID CONTROLLLER}

For stable and efficient rough terrain traversal, the robot's locomotion parameters are adapted to the structure of the terrain it is maneuvering on. In the proposed hybrid control architecture shown in Fig. 2, two approaches are combined to achieve the locomotion adaptation: reactive and proactive control [5], [6], [7]. The stride trajectory generator, impedance controller and inclination controller allow reactive ground interaction by adapting the legs' position and orientation based on proprioceptive sensing. This is complemented by a proactive approach to improve the maneuverability of Weaver. The main contribution of this work consists of the adaptation controller that uses exteroceptive sensing to adapt the gait parameters of the reactive controller and the virtual stiffness of the impedance controller. In addition, the reactive controller is augmented with a navigation system (i.e. visualinertial odometry and path follower) that focuses on a highlevel abstraction of the path to extend Weaver's autonomy. This allows Weaver to perform autonomous navigation in an unknown environment. The described hybrid controller feeds in to a hierarchical control architecture with three abstraction layers; 1.) the path follower in world frame, 2.) stride trajectory generator in body frame and 3.) impedance controller in leg frame. The following subsections describe the functional blocks depicted in Fig. 2.

\section{A. Visual-Inertial Odometry}

The proactive adaptation controller and the path follower in Fig. 2 require the robot's pose with respect to a fixed world frame $\left(o_{0} x_{0} y_{0} z_{0}\right)$. OKVIS, an open-source, keyframe based visual-inertial odometry algorithm is used for this purpose [14], [15]. The choice for visual-inertial odometry as a localization tool is motivated by the availability of the required sensing modalities (stereo camera and IMU) onboard the robot (Fig. 2). OKVIS uses nonlinear optimization procedures for tightly coupled fusion of visual and inertial measurements. The custom built sensor unit in this work consists of a rigid mount with two cameras and an IMU. The pitch angle of the camera mount is customized with a lockable revolute joint for adjustable capture of the scenery. KALIBR calibration package with an Aprilgrid is used for spatial calibration of this setup[16]. Triggering is synchronized via a GPIO cable connecting the cameras.

To the best of the authors' knowledge, this is the first instance of OKVIS being used on a hardware setup other than the fully time-synchronized and factory-calibrated VI-sensor [17] it was designed for. In contrast to reported experiments, where the sensor unit is either hand held or mounted on a car [15], the camera pair is tilted towards the ground since the terrain perception requires this visual angle. This leads to a field of view limited to objects relatively close to the cameras, resulting in a limited number of detectable features in the image streams. When maneuvering on uneven terrain, motion tends to be bumpy which further influences the performance of the algorithm.

\section{B. Path Follower}

Weaver's low level autonomy enables it to maneuver in rough terrain environments without using path planning of

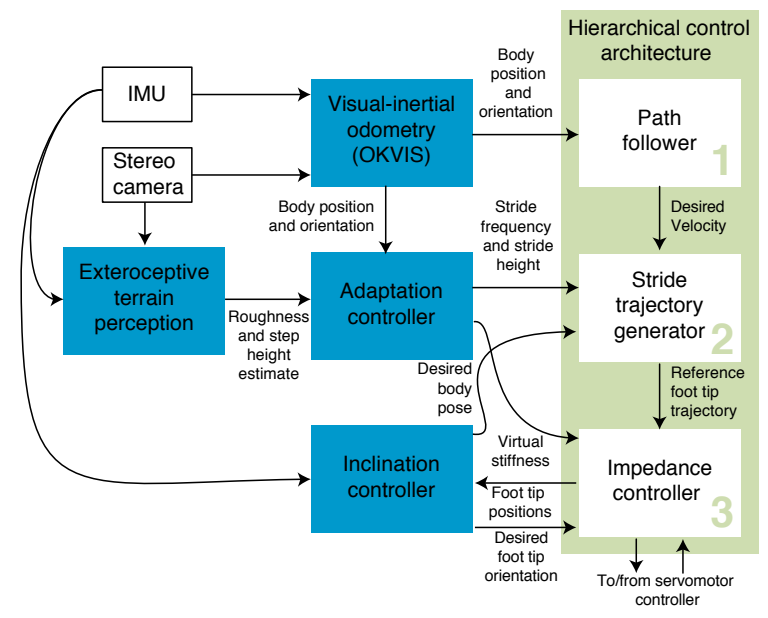

Fig. 2. Hybrid control architecture of Weaver. 
the individual foot tip positions [9]. Therefore, it suffices to control the movement of the body frame $\left(o_{1} x_{1} y_{1} z_{1}\right)$ with respect to a world frame $\left(o_{0} x_{0} y_{0} z_{0}\right)$ (Fig. 3). The world frame is defined as the initial body frame. Based on the position $o_{1}$ and orientation (yaw angle) $q_{y a w}$ of the body, the following algorithms derive the desired linear velocity vector $\left[\begin{array}{ll}V_{x} & V_{y}\end{array}\right]^{T}$ and rotational velocity $\dot{q}_{y a w}$ with respect to the body frame. The lookahead-based steering law based on straight line segments between waypoints is used for autonomous underwater vehicles [18]. This approach is adapted to the 2-D maneuvering problem of an omnidirectional hexapod robot.

Two waypoints $p_{k}$ and $p_{k+1}$ implicitly define the straightline path of the robot. The absolute speed of the robot is defined by $|V|=\sqrt{V_{x}^{2}+V_{y}^{2}}$ and the forward speed command $V_{x}$ is given by

$$
V_{x}=V_{x, \operatorname{Max}} \cdot \frac{s}{\sqrt{s^{2}+d^{2}}}
$$

where $s, d$, and $V_{x, \text { Max }}$ are the along-track distance, the lookahead distance and the maximum along-track speed respectively. The lookahead distance is given by $d=$ $\sqrt{R^{2}-e^{2}}$ where $R$ and $e$ are the radius of the circle of acceptance around the robot's position and the cross-track error, respectively. On uneven terrain, in particular, it is beneficial to use the sideways motion of the hexapod to minimize the cross-track error $e$ since rough terrain induces random disturbances due to slippage and moving objects on the surface. The desired sideways motion is

$$
V_{y}=\left\{\begin{array}{l}
V_{y, \operatorname{Max}} \cdot \frac{e}{\left|\alpha_{e}\right|} \text { if }\left|\alpha_{e}\right|<\alpha_{e 0} \\
0 \quad \text { if }\left|\alpha_{e}\right| \geq \alpha_{e 0}
\end{array}\right.
$$

where

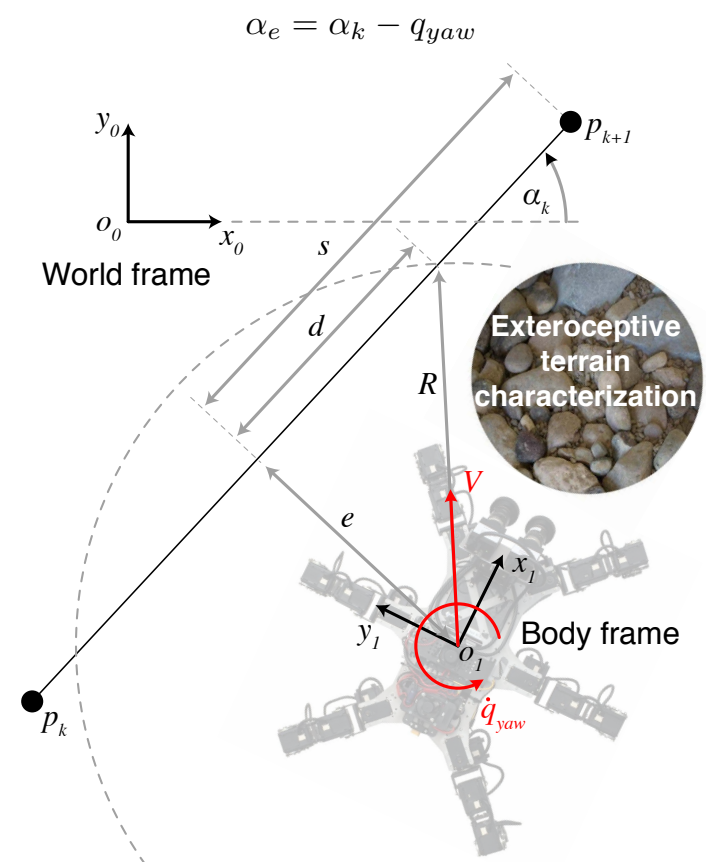

Fig. 3. Lookahead-based steering on straight line segments between waypoints.
The angle error between the straight-line orientation $\alpha_{k}$ and the orientation of the robot (yaw angle) $q_{y a w}$ is given by $\alpha_{e} . V_{y, \operatorname{Max}}$ and $\alpha_{e 0}$ are tuning parameters that specify the maximum sideways speed and the range of $\alpha_{e}$ for which the sideways motion is enabled. The desired rotational velocity centered at the vertical body axis $z_{2}$ is given by

$$
\dot{q}_{y a w}=-K_{\text {rot }} \cdot\left(\alpha_{d}-q_{y a w}\right)
$$

where

$$
\alpha_{d}=\alpha_{k}+\arctan \left(-\frac{e}{d}\right)
$$

$K_{\text {rot }}$ and $\alpha_{d}$ are the P-Gain of the heading autopilot and the desired course angle, respectively.

A switching mechanism selects the next line segment if the waypoint $p_{k+1}$ falls inside of the circle of acceptance with radius $R$.

\section{Stride Trajectory Generator, Impedance Controller and Inclination Controller}

The following subsection briefly discusses the stride trajectory generator, impedance controller and inclination controller displayed in Fig. 2. An in-depth analysis of these can be found in [9].

The stride trajectory generator creates a gait pattern for all six legs with a foot trajectory in the form of a Bézier curve. This is based on the velocity inputs from the global path follower, i.e. the gait pattern and foot trajectory are defined relative to the body frame $\left(o_{1} x_{1} y_{1} z_{1}\right)$. In this context, a stride describes the combined movement of the swing and stance phase.

The body velocity is a function of stride length $S_{\text {Stride }}$ and stride frequency $f_{\text {Stride }}$. In most of the documented work on legged locomotion just one of the two parameters is modified to control the speed of the robot [19]. In this work both parameters are modified by different control instances. The velocity command generated by the path follower sets the stride length $S_{\text {Stride }}$ and the adaptation controller changes the stride frequency $f_{\text {Stride }}$ based on the exteroceptive terrain perception. This allows a low stride frequency and a high stride length in rough terrain. Thus, the robot is able to maintain its stability due to the low stride frequency while the high stride length allows the robot to overcome challenging obstacles. Furthermore, the stride height $h_{\text {Stride }}$ obtained from the adaptation controller determines the height of the generated foot tip trajectory (Fig.2).

The impedance controller of the foot tip in Cartesian space reacts to the shape of the terrain by transforming the impact force at the foot tip into a resulting position. The second order mechanical system is given by

$$
-F_{z}=m_{v i r t} \Delta \ddot{z}_{r 2}+b_{v i r t} \Delta \dot{z}_{r 2}+c_{v i r t} \Delta z_{r 2}
$$

where $F_{z}, \Delta z_{r 2}, m_{v i r t}, b_{v i r t}$ and $c_{v i r t}$ are the force in vertical direction, the adapted position in vertical direction, the virtual mass, the virtual damping and the virtual stiffness, respectively. As described in the following sections, $c_{v i r t}$ is generated by the adaptation controller based on the perceived terrain features. The reference foot trajectory $z_{r 2}$ of the 
stride trajectory generator is adapted by $z_{d 2}=z_{r 2}-\Delta z_{r 2}$. Weaver with its novel 5 DoF leg design is able to control the Cartesian position and orientation of the foot tip. The last link of the leg is aligned with the gravity vector using inverse kinematics, i.e. the force ellipsoid of the foot tip is aligned with the gravity vector. Thus, the gravitational force of the robot is supported with the least amount of effort [20]. The inverse kinematics transforms the desired foot tip position $\left[\begin{array}{lll}x_{d 2} & y_{d 2} & z_{d 2}\end{array}\right]^{T}$ and orientation $\left[\begin{array}{ll}\delta_{d} & \beta_{d}\end{array}\right]^{T}$ into desired motor positions.

The inclination controller determines the orientation of the gravity vector with respect to the body frame $\left(o_{1} x_{1} y_{1} z_{1}\right)$ based on the IMU signals and the six foot tip positions, i.e. the orientation of the gravity vector is described by two angles $\delta_{d}$ and $\beta_{d}$ that constrain the space of solutions of the inverse kinematics. In addition, the inclination controller shifts the center of mass (CoM) of the robot on inclined terrain to increase stability.

\section{Exteroceptive Terrain Perception}

Visual-inertial odometry uses the stereo camera and the IMU to generate the pose of the robot with respect to the world frame $\left(o_{0} x_{0} y_{0} z_{0}\right)$. The stereo camera and the IMU are further used to characterize the terrain in front of the robot. This analysis serves as input for the adaptation controller. The following section is a summary of the work conducted in [13]. This work contains a detailed description of the applied stereo vision system and terrain feature extraction.

The generated point cloud in 3D space which is generated from the stereo image streams is aligned with the gravity vector based on the IMU. This allows for terrain intrinsic feature extraction [21]. In order to represent terrain elevation by a Digital Elevation Model (DEM), the horizontal plane is discretized into quadratic cells. The maximum terrain elevation within each cell is marked by a DEM point [22]. A plane is then fitted to the DEM points using a least squares method. From this plane and the elevation data of the DEM, a set of features $f_{i}$ are extracted from a terrain patch, approximately the size of the robot's footprint, in front of the robot (Fig. 4).

From subsets of the extracted features $f_{i}$ descriptive terrain characterization parameters, roughness $r_{a}$ and step height $h_{a}$, are derived. They serve as input for the adaptation controller. Fig. 4 summarizes the overall terrain perception procedure. The roughness characterization transforms the extracted features $f_{i}$ into a single estimate of the surface roughness $r_{a}$. The mathematical expression is given by

$$
r_{a}=\frac{1}{a_{n o r m, 1}} \sum_{i=1}^{5} a_{i} \cdot f_{i}
$$

and the step height $h_{a}$ is calculated by

$$
h_{a}=\frac{1}{a_{n o r m, 2}}\left(a_{6} \cdot f_{6}+a_{7} \cdot f_{4} \cdot f_{7}\right)
$$

The weighting parameters $a_{i}$ used to calculate the roughness $r_{a}$ and step height $h_{a}$ estimation are set empirically, i.e. generating suitable $r_{a}$ and $h_{a}$ for a number of example

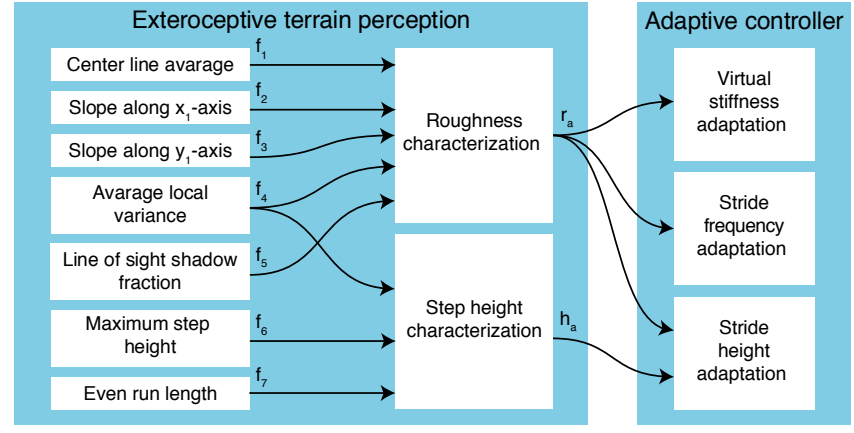

Fig. 4. Exteroceptive terrain perception diagram.

surface types. In addition, $r_{a}$ and $h_{a}$ are normalized by $a_{\text {norm, } i}$ between zero (i.e. even terrain, low step height) and one (i.e. uneven terrain, high step height). In the next section these generalized terrain characterization parameters are mapped into physical adaptation parameters.

\section{E. Vision-based Controller Adaptation}

Increasing or respectively decreasing the virtual stiffness $c_{v i r t}$, stride frequency $f_{\text {Stride }}$ and stride height $h_{\text {Stride }}$ leads to more difficulties during rough terrain traversal. Often the robot gets stuck and is unable to proceed at all. On flat terrain high virtual stiffness, high stride frequency and low stride height allow smooth and efficient locomotion, whereas the energy efficiency and velocity decreases for a different set of parameters. The following controller is designed for adaptation of the gait parameters and the impedance controller based on the exteroceptive terrain characterization parameters $r_{a}$ and $h_{a}$.

Similarly to the terrain characterization, the stiffness adaptation in Fig. 4 derives a suitable mapping between roughness $r_{a}$ and the virtual stiffness $c_{v i r t}$ in (6) of the impedance controller. The stiffness adaptation is defined as

$$
c_{v i r t}=b_{0}+b_{1} \cdot r_{a}+b_{2} \cdot r_{a}^{2}+b_{3} \cdot r_{a}^{3}
$$

The stride frequency $f_{\text {Stride }}$ and stride height $h_{\text {Stride }}$ of the stride trajectory generator are adapted by

$$
\begin{gathered}
f_{\text {Stride }}=c_{0}+c_{1} \cdot r_{a}+c_{2} \cdot r_{a}^{2} \\
h_{\text {Stride }}=d_{0}+d_{1} \cdot h_{a}+d_{2} \cdot r_{a}
\end{gathered}
$$

The parameters $b_{i}, c_{i}$ and $d_{i}$ are the coefficients of polynomial functions. Moreover, the polynomial functions are derived empirically for a number of exemplary terrain types. By this means, the roughness $r_{a}$ and step height $h_{a}$ are measured for different terrain structures. In addition, a desired (optimized with respect to locomotion speed) virtual stiffness $c_{v i r t}$, stride frequency $f_{\text {Stride }}$ and stride height $h_{\text {Stride }}$ are chosen for these terrains. Polynomial functions are fitted to the data points (Fig. 5). A least square fit gives the coefficients and degree of the polynomials in (9), (10) and (11).

As the robot perceives terrain features at a given distance in front, information on ego-motion is needed (Fig. 3). The body trajectory and orientation given by the visual-inertial odometry are used to update a spatial map of roughness and 


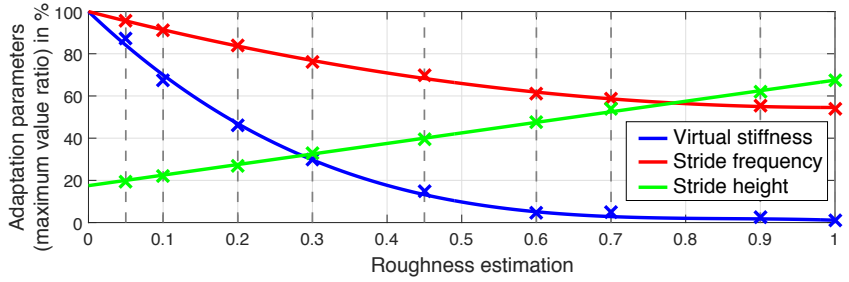

Fig. 5. Polynomial function fit for nine exemplary terrain types from the multi-terrain testbed.

step height values in the world frame. A circular area around the center of the body frame which is relevant for the robot's foot tip placement is searched for the highest roughness and step height values. Thus, the most conservative set of gait parameters is set so that the robot can safely traverse the given area.

\section{EXPERIMENTS AND RESULTS}

\section{A. Performance Criteria}

The dimensionless energetic cost of transport (CoT) is a popular performance indicator for wheeled and legged robots. For more information about the cost of transport please refer to [9], [23]. The instantaneous cost of transport $C o T$ and the overall cost of transport over a travelled distance $\overline{C o T}$ are given by

$$
C o T=\frac{U I}{m g v}, \overline{C o T}=\frac{\frac{1}{n} \sum_{i=1}^{n} U_{i} I_{i}}{m g \frac{\Delta x}{\Delta t}},
$$

where $U$ is the voltage of the power supply, $I$ is the instantaneous current drawn from the power supply, $m$ is the mass, $g$ is the gravitational acceleration, $v$ is the velocity of the robot and $\Delta t$ is the time needed for the travelled distance $\Delta x$. The CoT in this work captures the overall energy consumption of the robot based on the voltage and current of the power supply. This includes mechanical energy, heat dissipation and other losses like friction. The energy efficiency highly depends on the characteristics of the servomotors (e.g. PID gains, resistance, induction, reduction ratios). Since this work compares the CoT of two control algorithms on the same robotic platform, it is assumed that the influence of these characteristics remain unchanged between evaluation runs.

The percentage reduction of variance $S_{i}$ comes from ship control assessment [24] and it is given by

$$
S_{i}=100 \cdot\left(1-\frac{\operatorname{Var}\left(X_{i, a}\right)}{\operatorname{Var}\left(X_{i, n a}\right)}\right)
$$

where $i$ is a place holder for the movement in pitch and roll. The function $\operatorname{Var}(\cdot)$ is the variance and the variable $X_{i}$ is the set of observed values in radiant. The subscripts $a$ and $n a$ stand for adaptive and non-adaptive, respectively. Maximizing (13) leads to stable movement of the body.

\section{B. Experimental Setup}

As discussed in Section III, Weaver has no a priory information about its environment and no exteroceptive sensors were used for path planning of the foot tips. All computation

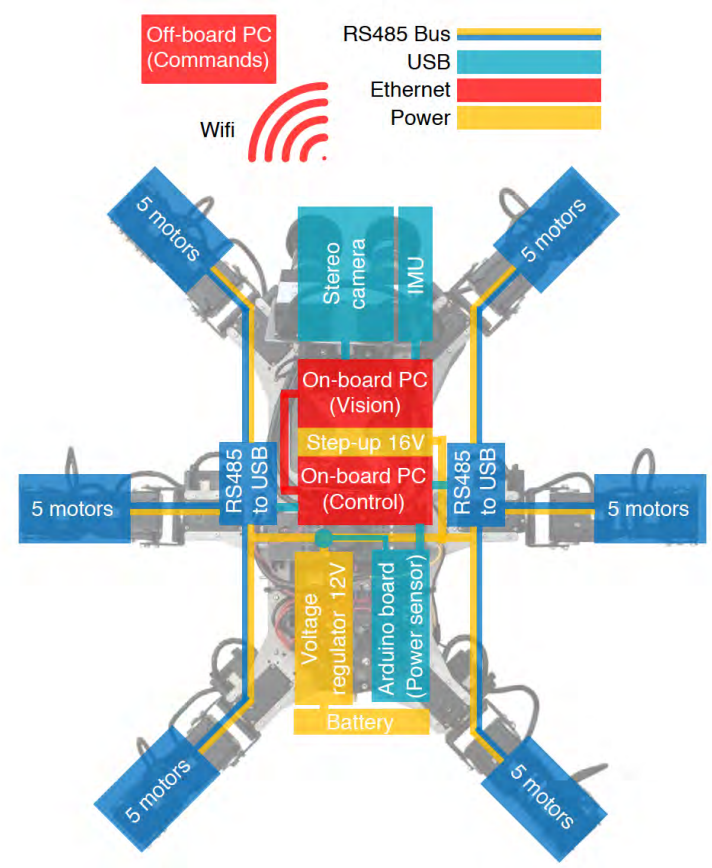

Fig. 6. Hardware structure of Weaver.

is processed on-board and online using two mini PCs. A human operator specifies a desired waypoint for the robot to reach via an off-board PC (connected via WiFi). The robot used a tripod gait during experimentation. As the robot is powered by battery, it does not require any external wires to operate. Weaver operates for approximately $1 \mathrm{hr}$ with the current hardware configuration. Figure 6 shows the overall structure of Weaver during experimentation and Table I summarizes the main specifications of Weaver.

The CoT in (12) requires the measurement of the robot's velocity $v$ and overall power consumption $P$. Therefore, the velocity $v$ of the robot was tracked at $4 \mathrm{~Hz}$ using a robotic total station (Leica TS12) with a target prism mounted on the robot. The power consumption $P$ was measured at $20 \mathrm{~Hz}$ by an Arduino based system sensor. The range of the adaptive parameters and the constant values of the nonadaptive approach are summarized in Table II.

To generate comparable experimental results, a multiterrain testbed in Fig. 7 was built. The robot starts on flat ground (segment $\mathrm{A}$ ) and then passes an inclined plane (segment $\mathrm{B}$ ) before entering the rough terrain (segments $\mathrm{C}$ to F). Segment $C$ of the testbed contains wooden blocks of various heights and segment D-E-F is a mixture of sand,

TABLE I

HARDWARE SPECIFICATIONS OF WEAVER.

\begin{tabular}{cl}
\hline Type & Description \\
\hline General & Mass: 9.3 kg (without battery), 10.3 kg (with battery) \\
Servomotors & Dynamixel MX-64 and MX-106 \\
Power supply & 7-cell LiPo battery (25.9 V, 5000 mAh) \\
On-board PC & $2 \times$ Intel NUC mini PC (Intel Core i7 processor, \\
& 16 GB RAM) running Robot Operation System (ROS) \\
& in an Ubuntu environment \\
IMU (Microstrain GX3 $-100 \mathrm{~Hz})$ and $2 \times$ Cameras \\
& (Pointgrey Grasshopper3) \\
\hline
\end{tabular}




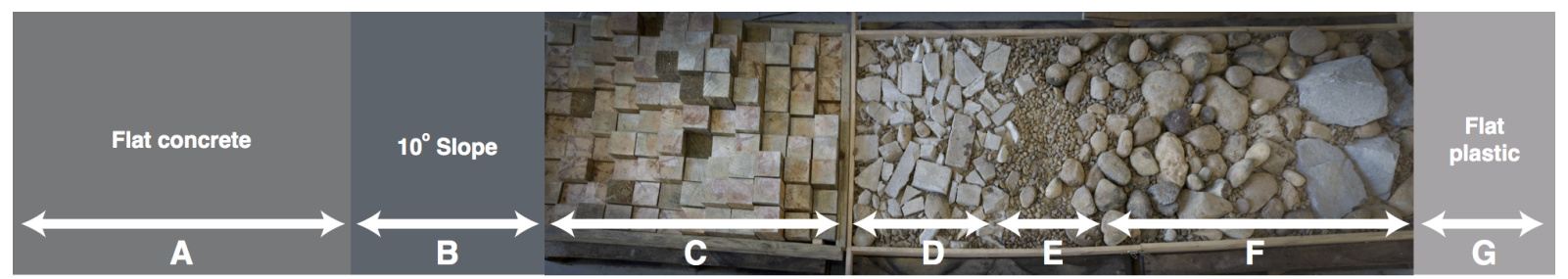

Fig. 7. Multi-terrain testbed with maximum height difference: $113 \%$ (segment C), $28 \%$ (segment D), $11 \%$ (segment E) and $72 \%$ (segment F) of Weaver's body height. $2.93 \mathrm{~m}$ of flat ground (segment A) followed by $1.2 \mathrm{~m}$ of inclined planar section $\left(10^{\circ}\right)$ (segment B) are traversed before entering segment C. Weaver finishes its run on flat ground (segment $\mathrm{G})$. The width of the testbed is $145 \%$ of Weaver's start-up width.

pebbles, river stones, crumbled concrete and bigger stones.

\section{Results}

The first experiment evaluates the performance of OKVIS and the path follower. Fig. 8 shows the behavior of Weaver on flat terrain by following a pattern of straight lines (black dashed line) which are implicitly defined by five waypoints. The path follower uses the position and orientation signals of OKVIS to navigate on the desired path. It can be seen that the path follower reduces the position error between the robot's position given by OKVIS (blue line) and desired path (black/dashed line). The error at the waypoints originates from the switching mechanism described in Section III-B. Here, the radius $R$ of the circle of acceptance is $0.5 \mathrm{~m}$. In this experiment, the position returned by the Leica system is used as ground truth for the robot's position. The OKVIS position error, being the difference between this ground truth (red line) and OKVIS position (blue line) increases as the robot moves along the desired path. It can be noticed that the odometer mainly drifts in the direction of travel. Therefore, the real position of the robot deviates from the desired path (black/dashed line). The final position error on flat terrain is $0.64 \mathrm{~m}$ for a travelled distance of $20.94 \mathrm{~m}$. Since the robot is supposed to move on rough terrain, Fig. 9 evaluates the performance of OKVIS on the mulit-terrain testbed of Fig. 7. Weaver walked 30 times over the testbed with its navigation system. It can be seen that the testbed induces drift mainly in the forward direction ( $x_{1}$ direction) of Weaver (upper plot). This enables the robot to navigate through the narrow testbed since the drift in the side ways direction ( $y_{1}$ direction) of Weaver is negligible. Moreover, three standard deviations of the robot's path over 30 runs stays inside of the testbed (middle plot). The lower plot shows how the OKVIS error propagates over the testbed and the mean of the total error in the end of the testbed is $0.36 \mathrm{~m}$.

These 30 autonomous runs were also used to evaluate the performance of the adaptation controller on the multi-

TABLE II

RANGE OF THE ADAPTIVE PARAMETERS AND CONSTANT VALUE OF THE NON-ADAPTIVE CONTROLLER.

\begin{tabular}{lccc}
\hline Adaptive parameters & Min value & Max value & Const. value \\
\hline Virtual stiffness $\left(\mathrm{Nm}^{-1}\right)$ & $0.7510^{3}$ & $70.3410^{3}$ & $1.0610^{3}$ \\
Stride frequency $(\mathrm{Hz})$ & 0.6 & 1.1 & 0.6 \\
Stride height $(\mathrm{m})$ & $2.3110^{-2}$ & $13.1810^{-2}$ & $9.8910^{-2}$ \\
\hline
\end{tabular}

terrain testbed. Two sets of 15 runs have been conducted to compare the CoT using adaptive against non-adaptive control as a reference. These are referred to as adaptive set and non-adaptive set respectively. The parameter set of the non-adaptive controller is tuned to match the locomotion behaviour of the adaptive controller on the roughest terrain part (segments C to F). As can be seen in [9], a nonadaptive controller tuned to match the locomotion of the adaptation controller on flat terrain (segment A) is incapable of finishing the terrain task. Fig. 10 shows the CoT (upper figure) and the corresponding adaptation parameters (lower figure). The lines depict the mean CoT of the 15 runs whereas the grey shading visualizes one standard deviation of the CoT of the adaptive set. The difference in CoT in segments A and B can be explained by angular and vertical robot body motion which occurs when walking on even ground with a low stiffness. Additional factors for the reduction of energy consumption are a higher stride frequency as well as reduced stride height, as flat terrain can be traversed without excessively lifting the legs. The rough terrain of segment $\mathrm{C}, \mathrm{E}$ and $\mathrm{F}$ shows just a small reduction of CoT. This is as expected since almost equal gait parameters are applied in both sets. In segment D, the adaptive approach has clear advantages compared to the non-adaptive set. High CoT values in the non-adaptive set imply that the robot has difficulties in traversing the terrain. Table III summarizes the CoT reduction for each segment of the multi-terrain testbed. The overall CoT over the multi-terrain testbed are $36.43 \pm 1.68$ (adaptive set) and $51.74 \pm 3.46$ (non-adaptive set). Therefore, the adaptive controller reduces the overall CoT by $29.59 \%$. On flat terrain the minimum CoT of the

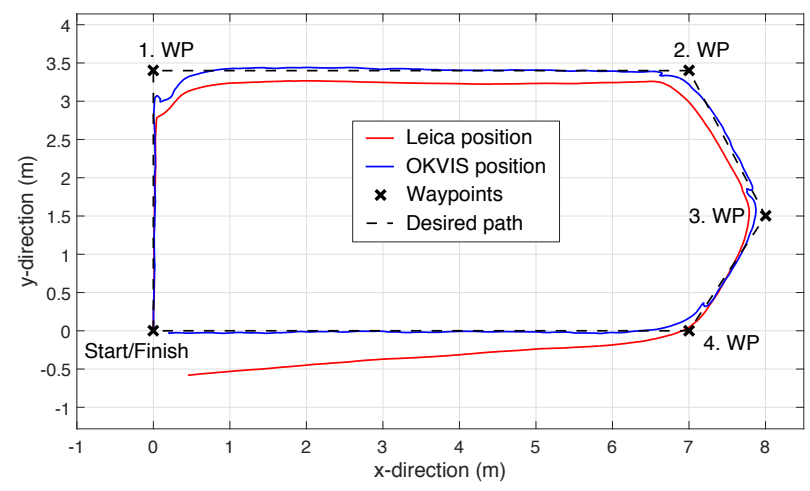

Fig. 8. Autonomous path following of five waypoints on flat terrain showing the behavior of OKVIS and the path follower. The ground truth is considered to be the position returned by the Leica system. 

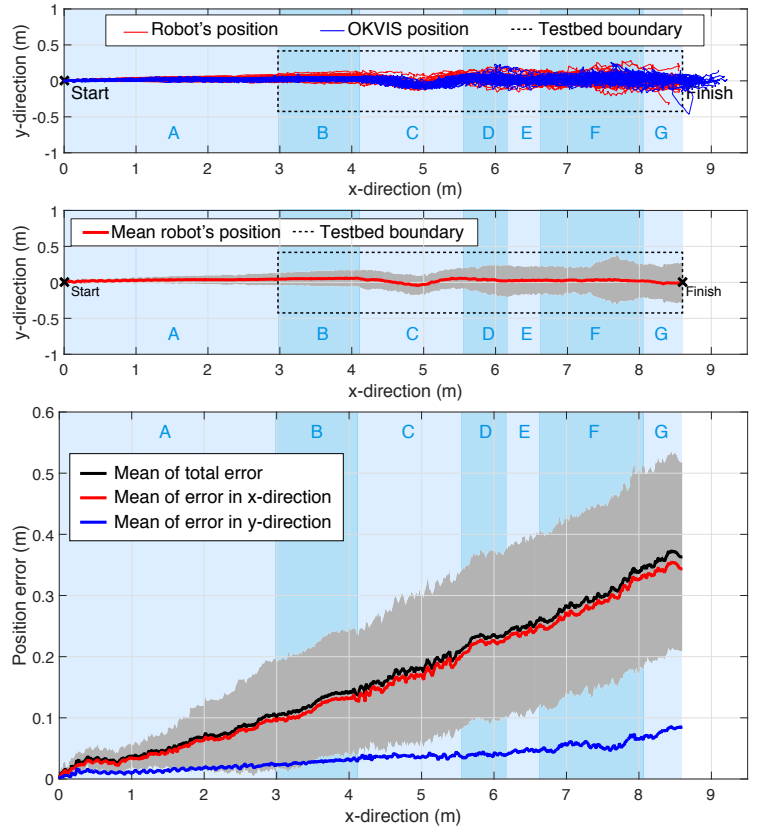

Fig. 9. Walking 30-times autonomously over the multi-terrain testbed: The upper figure shows the robot and OKVIS position of all runs. The figure in the middle shows the mean of robot positions (red line) and three standard deviations of robot's path (grey shading). The means of the total error (black line), error in x-direction (red line) and error in y-direction (blue line) between robot's and OKVIS position is presented in the lower figure. One standard deviation of the total error is given by the grey shading.

adaptive controller is approximately 10 and the maximum velocity is $0.35 \mathrm{~m} / \mathrm{s}$.

The percentage reduction of variance (13) in Table III is calculated as the mean of the 15 runs of each set. The roll and pitch movement is distinctly reduced in segment A. On the inclined terrain (segment B) the variance of the pitch movement of the adaptive controller is greater than the variance of the non-adaptive approach. This can be explained by considering the right plot of Fig. 11. The pitch angle of the non-adaptive approach increases already on flat terrain (segment A) due to the low virtual stiffness of each leg. Thus, on the inclined terrain (segment B) the pitch angle does not increase as much as the pitch angle of the adaptive approach. This also explains the negative $S_{\text {pitch }}$ value in segment C. For the remaining part of the testbed (segment $\mathrm{C}$ to $\mathrm{F}$ ) it can be seen that on average the angular movement is reduced by the adaptation controller.

TABLE III

COMPARISON OF PERCENTAGE REDUCTION IN VARIANCE OF COT FOR MULTI-TERRAIN TESTBED SEGMENTS.

\begin{tabular}{cccc}
\hline $\begin{array}{c}\text { Segments of } \\
\text { the testbed }\end{array}$ & $\begin{array}{c}\text { CoT reduction } \\
\text { in } \%\end{array}$ & $\begin{array}{c}S_{\text {roll }} \\
\text { in } \%\end{array}$ & $\begin{array}{c}S_{\text {pitch }} \\
\text { in \% }\end{array}$ \\
\hline A & 65.82 & 81.41 & 87.70 \\
B & 36.93 & 34.86 & -155.26 \\
C & 12.87 & 2.4 & -23.00 \\
D & 27.11 & -2.28 & 22.91 \\
E & 15.76 & 13.85 & 53.91 \\
F & 16.01 & 3.77 & 3.50 \\
\hline
\end{tabular}
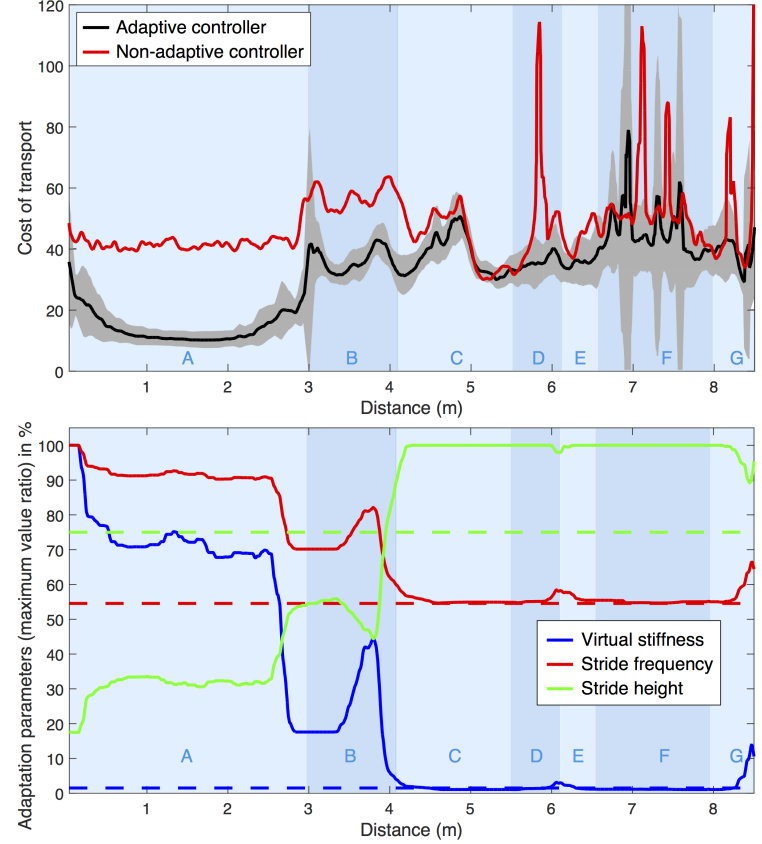

Fig. 10. The CoT of the adaptive and non-adaptive controller show the results of 15 runs each on the multi-terrain testbed. Upper figure: The black line and the grey shading are the mean and one standard deviation of the CoT of the adaptive set. The red line shows the mean of the CoT of the nonadaptive set. Lower figure: The percentage values of adaptation parameters are shown. The solid lines are the mean parameters of the adaptive set and the dashed lines show the values of the non-adaptive approach.

\section{Discussion}

The results demonstrate that Weaver is able to autonomously overcome challenging terrain with the adaptive as well as with the non-adaptive approach. Nevertheless, adaptive control significantly reduces the CoT compared to the non-adaptive approach and improves the body stability by reducing the angular movement in roll and pitch. The hybrid controller adapts the impedance controller complemented by adaptation of stride frequency and stride height. The adaptation method is based on online exteroceptive terrain perception and thus, does not require any prior information of the environment. This control architecture solves the problem of conflicting objectives when traversing different terrain structures, e.g. transition from even to uneven terrain. Moreover, Weaver automatically chooses optimized parameters of the reactive controller based on proactive terrain analysis. The results of the odometry evaluation (Section IV-C) shows that

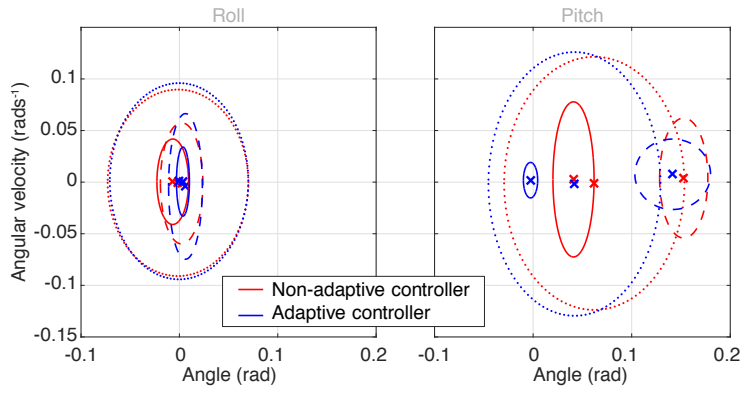

Fig. 11. Mean (x) and one standard deviation (ellipse) of the limit cycles of the roll and pitch movement projected onto the phase plane during traversal on the multi-terrain testbed. Solid, dashed and dotted line refers to segment $\mathrm{A}, \mathrm{B}$ and $\mathrm{C}$ to $\mathrm{F}$, respectively. 
the robot is able to autonomously reach a given waypoint. Odometer drift mainly depends on detectable features in the camera images and on the robot's motion characteristics. Global navigation accuracy is limited by the accuracy of the odometer. However, the drift of the spatial translation from the perception location to the robot's location which is needed by the adaptation controller (see Section III-E) is negligible due to the small travelled distance $(<1 \mathrm{~m})$. The global navigation system for omnidirectional vehicles combined with adaptive control increases the autonomy of Weaver on rough terrain. The robot adapts its speed by controlling the stride length and stride frequency simultaneously, i.e. the stride length is set by the path follower and the stride frequency changes based on terrain perception. As shown in Fig. 7, the testbed is rather narrow with respect to the robot's walking width. The path follower effectively reduces the error between the desired path and the robot's trajectory and therefore prevents the robot from stepping out of the testbed during experimentation.

\section{CONCLUSIONS}

This work presented a hybrid control architecture for the Weaver hexapod, combining reactive and proactive control paradigms to enhance the performance in rough terrain in terms of reduced CoT and increased body stability. Weaver's controller adapts the locomotion parameters using stereovision based perception of the terrain. In addition, this work extends the control architecture with a navigation system that uses a high-level abstraction of the robot's path. The reactive controller complemented with the adaptive controller handles the low-level autonomy on rough terrain. This avoids the use of complex planning algorithms based on detailed maps of the environment. The experimental results illustrate that Weaver is capable of autonomously maneuvering on uneven terrain in an effective manner. Moreover, Weaver's current configuration is self-contained in terms of processing and energy (i.e. tether free) making it suitable for fully autonomous operation. This lends itself to applications such as short distance exploration tasks in remote and challenging environments, extending the scope and utility of legged robots. However, stereo vision may lead to wrong estimations from conditions such as walking in high grass, bad illumination, motion blur, or dynamic scenes. The authors aim to address this by adding proprioceptive sensing in future work.

\section{ACKNOWLEDGEMENTS}

The authors would like to thank Ryan Steindl, Brett Wood and John Whitham for their support during the project. This work was fully funded by the CSIRO.

\section{REFERENCES}

[1] A. Howard, "Real-time stereo visual odometry for autonomous ground vehicles," in IEEE/RSJ International Conference on Intelligent Robots and Systems (IROS), 2008, pp. 3946-3952.

[2] T. Ohki, K. Nagatani, and K. Yoshida, "Path planning for mobile robot on rough terrain based on sparse transition cost propagation in extended elevation maps," in IEEE International Conference on Mechatronics and Automation (ICMA), 2013, pp. 494-499.
[3] G. Li, R. Song, C. Chen, Y. Li, and C. Zhang, "The traversability analysis for coal mine mobile robot based on rough sets," in IEEE International Conference on Robotics and Biomimetics (ROBIO), 2013, pp. 420-424.

[4] K. Hauser, T. Bretl, J.-C. Latombe, K. Harada, and B. Wilcox, "Motion planning for legged robots on varied terrain," The International Journal of Robotics Research, vol. 27, no. 11-12, pp. 1325-1349, 2008.

[5] A. Roennau, G. Heppner, M. Nowicki, J. Zoellner, and R. Dillmann, "Reactive posture behaviors for stable legged locomotion over steep inclines and large obstacles," in IEEE/RSJ International Conference on Intelligent Robots and Systems (IROS), 2014, pp. 4888-4894.

[6] R. C. Arkin, Behavior-based robotics. MIT press, 1998.

[7] J. P. Müller, "Control architectures for autonomous and interacting agents: A survey," in Intelligent Agent Systems Theoretical and Practical Issues. Springer, 1997, pp. 1-26.

[8] D. Wettergreen, H. Pangels, and J. Bares, "Behavior-based gait execution for the dante ii walking robot," in IEEE/RSJ International Conference on Intelligent Robots and Systems (IROS), 1995, pp. 274 279.

[9] M. Bjelonic, N. Kottege, and P. Beckerle, "Proprioceptive control of an over-actuated hexapod robot in unstructured terrain," in IEEE/RSJ International Conference on Intelligent Robots and Systems (IROS), 2016, pp. 2042-2049.

[10] J. K. Hodgins and M. Raibert, "Adjusting step length for rough terrain locomotion," IEEE Transactions on Robotics and Automation, pp. 289-298, 1991.

[11] Y. Fukuoka, H. Kimura, and A. H. Cohen, "Adaptive dynamic walking of a quadruped robot on irregular terrain based on biological concepts," The International Journal of Robotics Research, pp. 187-202, 2003.

[12] S. Hirose, H. Tsukagoshi, and K. Yoneda, "Normalized energy stability margin and its contour of walking vehicles on rough terrain," in IEEE International Conference on Robotics and Automation (ICRA), 2001, pp. 181-186.

[13] T. Homberger, M. Bjelonic, N. Kottege, and P. V. K. Borges, "Terraindependent motion adaptation for hexapod robots," in International Symposium on Experimental Robotics (ISER), 2016.

[14] S. Leutenegger, S. Lynen, M. Bosse, R. Siegwart, and P. Furgale, "Keyframe-based visualinertial odometry using nonlinear optimization," The International Journal of Robotics Research, vol. 34, pp. 314-334, 2014.

[15] S. Leutenegger, P. Furgale, V. Rabaud, M. Chli, K. Konolige, and R. Siegwart, "Keyframe-based visual-inertial slam using nonlinear optimization," in Robotics: Science and Systems (RSS), 2013.

[16] P. Furgale, J. Rehder, and R. Siegwart, "Unified temporal and spatial calibration for multi-sensor systems," in IEEE/RSJ International Conference on Intelligent Robots and Systems (IROS), 2013, pp. 12801286.

[17] J. Nikolic, J. Rehder, M. Burri, P. Gohl, S. Leutenegger, P. T. Furgale, and R. Siegwart, "A synchronized visual-inertial sensor system with fpga pre-processing for accurate real-time slam," in IEEE International Conference on Robotics and Automation (ICRA), 2014, pp. 431-437.

[18] M. Breivik and T. I. Fossen, "Guidance laws for autonomous underwater vehicles," 2009.

[19] D. J. Hyun, S. Seok, J. Lee, and S. Kim, "High speed trotrunning: Implementation of a hierarchical controller using proprioceptive impedance control on the MIT Cheetah," The International Journal of Robotics Research, vol. 33, no. 11, pp. 1417-1445, 2014.

[20] M. Görner, T. Wimböck, A. Baumann, M. Fuchs, T. Bahls, M. Grebenstein, C. Borst, J. Butterfass, and G. Hirzinger, "The DLR-Crawler: A testbed for actively compliant hexapod walking based on the fingers of DLR-Hand II,' in IEEE/RSJ International Conference on Intelligent Robots and Systems (IROS), 2008, pp. 1525-1531.

[21] R. Hoffman and E. Krotkov, "Terrain roughness measurement from elevation maps," in Advances in Intelligent Robotics Systems Conference, 1990, pp. 104-114.

[22] R. Aeschimann and P. V. K. Borges, "Ground or obstacles? detecting clear paths in vehicle navigation," in IEEE International Conference on Robotics and Automation (ICRA), 2015, pp. 3927-3934.

[23] N. Kottege, C. Parkinson, P. Moghadam, A. Elfes, and S. P. N. Singh, "Energetics-informed hexapod gait transitions across terrains," in IEEE International Conference on Robotics and Automation (ICRA), 2015, pp. 5140-5147.

[24] T. Perez, "Ship motion performance," in Ship Motion Control, ser. Advances in Industrial Control. Springer London, 2005, pp. 127142 